\title{
Commutative morphic rings of stable range 2
}

\section{Oksana Pihura, Bohdan Zabavsky}

\begin{abstract}
It is know that a left quasi-morphic ring $R$ is a ring of stable range 1 if and only if $\operatorname{dim} R=0$. In this paper it is shown that a commutative morphic $\operatorname{ring} R$ is a ring of stable range 2 if and only if $\operatorname{dim} R=1$.
\end{abstract}

Keywords Stable range 1, stable range 2.

Mathematics Subject Classification (2000) 15S50, 16 U80

UDK 512.552 .13

\section{Introduction}

In his pioneering paper [3] Kaplansky has raised the question: If $a R=b R$ in a ring $R$, are $a$ and $b$ necessarily right associates? He remarked that for a commutative ring the property holds for the principal ideal rings and artinian rings.

Developing these ideas Canfell [2] to introduce the concept of the set of principal ideals $\left\{a_{i} R \mid i=1,2, \ldots, n\right\}$ is uniquely generated if whenever $a_{i} R=b_{i} R$ there exist elements $u_{i} \in R$ such that $a_{i}=b_{i} u_{i}, i=1,2, \ldots, n$, and $u_{1} R+u_{2} R+$ $\ldots+u_{n} R=R$. The dimension of a commutative $\operatorname{ring} R$, denoted by $\operatorname{dim} R$, is the least integer $n$ such that every set of $n+1$ principal ideals is uniquely generated. In [3] Canfell obtained characterizations of the $n$-dimensional $F$-spaces in terms of the rings of continuous real-valued and complex-valued functions defined on the space. By extending the notion of uniqueness of generators of principals he gave an algebraic characterization of the concept " $n$-dimensional".

In [1] Bass introduced the notion of stable range for a ring. A ring $R$ with stable range one is both left and right uniquely generated [6]. The converse does 
not hold true in general. Then $\mathbb{Z}$ is uniquely generated. Indeed, the ring $\mathbb{Z}$ is uniquely generated, but $\mathbb{Z}$ does not have stable range one. The stable range of $\mathbb{Z}$ is equal $2[6]$.

In the case of a left quasi morphic ring we have that property a left uniquely generated is equivalent to the property of stable range one [5].

In this article we will show that in the case of a commutative morphic ring we see that the property $\operatorname{dim} R=1$ is equivalent to the property of the stable range 2 .

\section{Results}

All our rings are commutative with identity. A ring $R$ is said to have stable range 2 if for any $a, b, c \in R$ such that $a R+b R+c R=R$ there exist $x, y \in R$ such that $(a+c x) R+(b+c y) R=R[6]$ (in the notion st.r $(R)=2$ ). We say that a ring $R$ is an almost Baer ring if for each $x \in R$ there exists an element $y \in R$ such that $\operatorname{Ann}(x R)=y R$ where $\operatorname{Ann}(x R)=\{z \mid z x r=0, r \in R\}$. By a Bezout ring we mean a ring in which all finitely generated ideals are principal. Two rectangular matrices $A$ and $B$ are equivalent if there exist invertible matrices $P$ and $Q$ of adequate size such that $B=P A Q$. A ring $R$ is Hermite if every rectangular matrix $A$ over $R$ is equivalent to an upper or a lover triangular matrix [3]. Any Hermite ring is a Bezout ring [2]. In [6] we have the following theorem.

Theorem 1 A commutative Bezout ring $R$ is an Hermite ring if and only if $\operatorname{st} . r(R)=2$.

A commutative ring $R$ is called a morphic ring if for any $a \in R$ there is an isomorphism $R / R a \cong \operatorname{Ann}(a)$ as of $R$ modules.

Theorem 2 Let $R$ be a commutative Bezout ring and $\operatorname{dim} R=1$. Then $\operatorname{st} . \mathrm{r}(R)=$ 2 .

Proof Let $a, b \in R$. Since $R$ is a commutative Bezout ring, $a R+b R=d R$, for some element $d \in R$. There exist $a_{0}, b_{0} \in R$ and $u, v \in R$ such that $a=d a_{0}$, $b=d b_{0}$ and $d=a u+b v=a_{0} u d+b_{0} v d$. Put $q=1-a_{0} u-b_{0} v$.

Then $d q=0$ and for any elements $t_{1}, t_{2} \in R$ we $\left(a_{0}+t_{1} q\right) d=a,\left(b_{0}+t_{2} q\right) d=b$. We will choose the $t_{i}, i=1,2$. So that the elements $a_{0}+t_{1} q=a_{1}$ and $b_{0}+t_{2} q=b_{2}$ generate $R$.

Then $a_{1} x+b_{1} y=1$ for some elements $x, y \in R$ and $a=a_{1} d, b=b_{1} d$. By [3] $R$ is an Hermite ring and by Theorem 1 we have $\operatorname{st} . r(R)=2$. The theorem is proved. 
Theorem 3 Let $R$ be a commutative almost Baer Bezout ring of stable range 2. Then $\operatorname{dim}(R)=1$.

Proof Let $a_{1} R=b_{1} R$ and $a_{2} R=b_{2} R$. Then $a_{1}=x_{1} b_{1}, a_{2}=x_{2} b_{2}$ and $b_{1}=y_{1} a_{1}$, $b_{2}=y_{2} a_{2}$ for some $x_{1}, x_{2}, y_{1}, y_{2} \in R$. Then $b_{1}\left(1-x_{1} y_{1}\right)=0, b_{2}\left(1-x_{2} y_{2}\right)=0$ and $1-x_{1} y_{1} \in \operatorname{Ann}\left(b_{1} R\right), 1-x_{2} y_{2} \in \operatorname{Ann}\left(b_{2} R\right)$. Let $\operatorname{Ann}\left(b_{1} R\right)=\alpha_{1} R$ and $\operatorname{Ann}\left(b_{2} R\right)=\alpha_{2} R$ for some $\alpha_{1}, \alpha_{2} \in R$.

Since $1-x_{1} y_{1} \in \alpha_{1} R$ and $1-x_{2} y_{2} \in \alpha_{2} R$, we have $x_{1} R+\alpha_{1} R=R$ and $x_{2} R+\alpha_{2} R=R$. Obviously, $x_{1} R+x_{2} R+\alpha_{1} \alpha_{2} R=R$. Since $\operatorname{st} . \mathrm{r}(R)=2$, we have $\left(x_{1}+\alpha_{1} \alpha_{2} s\right) R+\left(x_{2}+\alpha_{1} \alpha_{2} t\right) R=R$ for some $s t \in R$. Since $\left(x_{1}+\alpha_{1} \alpha_{2} t\right) b_{1}=$ $x_{1} b_{1}+\alpha_{2} t \alpha_{1} b=x_{1} b_{1}=a_{1},\left(x_{2}+\alpha_{1} \alpha_{2} s\right) b_{2}=x_{2} b_{2}+\alpha_{1} s \alpha_{2} b=x_{2} b_{2}=a_{2}$. Denote $x_{1}+\alpha_{1} \alpha_{2} t=u_{1}, x_{2}+\alpha_{1} \alpha_{2} s=u_{2}$.

We proved $u_{1} b_{1}=a_{1}, u_{2} b_{2}=a_{2}$ and $u_{1} R+u_{2} R=R$, that is $\operatorname{dim}(R)=1$. The theorem is proved.

A commutative morphic ring is an obvious example of an almost Baer Bezout ring [4].

As a consequence of Theorem 2 and Theorem 3 we obtain the following result.

Theorem 4 A commutative morphic ring $R$ is a ring of stable range 2 if and only if $\operatorname{dim}(R)=1$.

\section{References}

1. H. Bass K-theory and stable algebra. Inst. Hautess Etudes Sci. Publ.Math., 1964 22, 5-60.

2. M. J. Canfell Uniqueness of generators of principal ideals in rings of continuous function. Proc. Amer. Math. Soc., 1970 26, 517-573.

3. I. Kaplansky Elementary divisirs and modules. Trans. Amer. Math. Soc., 1949, 66, 464491.

4. W.K. Nicholson, E. Sanchez Campos Rings with the dual of the isomorphism theorem. J. Algebra, 2004 271, 391 - 406.

5. F. Siddique On two questions of Nicholson. arXiv: 1402.4706V1 [math. RA] 1S Feb 2014, 1-5.

6. B.V. Zabavsky Diagonal reduction of matrices over rings. Mathematical Studies, Monograph Series, v. XVI, VNTL Publishers, 2012, 251.

\section{Oksana Pihura}

Ivan Franko National University of Lviv,

1 Universytetska Str., 29000 Lviv

E-mail: pihuraoksana@mail.ru 


\section{Bohdan Zabavsky}

Ivan Franko National University of Lviv,

1 Universytetska Str., 29000 Lviv

E-mail: zabavskii@gmail.com 